\title{
Percepção de alunos sobre as expectativas do professor acerca do seu desempenho: um estudo comparativo entre alunos com e sem dificuldades de aprendizagem
}

\author{
Andreza Schiavoni \\ Selma de Cássia Martinelli \\ Universidade Estadual de Campinas
}

\begin{abstract}
RESUMO
A presente pesquisa teve como objetivo investigar se crianças com diferentes níveis de dificuldades de aprendizagem em escrita se diferem no que se refere à percepção de expectativas de seus professores a respeito delas. Participaram do estudo 138 crianças entre 9 e 10 anos de idade de duas escolas públicas. As dificuldades de aprendizagem em escrita foram avaliadas mediante uma escala padronizada, que detecta as dificuldades lingüísticas mais comuns na escrita de crianças das séries iniciais do ensino fundamental. A percepção dos participantes sobre as expectativas de seus professores a seu respeito foi obtida por meio de uma escala contendo vinte afirmações, onze positivas, que indicam boa percepção do aluno, e nove que indicam uma percepção negativa. Os resultados confirmaram a existência de diferenças entre a percepção de alunos sobre as expectativas a respeito deles, nos diferentes grupos de dificuldades de aprendizagem em escrita. As médias de percepção foram menores para os níveis mais altos de dificuldades e maiores para os grupos que apresentaram menos dificuldades de aprendizagem na escrita, indicando que quanto maior o nível de dificuldade de aprendizagem dos participantes, menos positiva se mostrou a percepção que têm sobre as expectativas dos professores a seu respeito.
\end{abstract}

Palavras-chave: percepção; expectativas de professores; dificuldades de aprendizagem.

\section{ABSTRACT \\ Students' perception about their teachers' expectancies towards their performance: a comparative study among students with and without learning difficulties}

This research aimed to investigate if children with different levels of difficulty in learning to write differ concerning the perception of their teacher's expectancies towards them. Participants were 138 children aged between 9 and 10 years from two public schools. A standardized scale was used to evaluate the difficulties in learning to write. This scale evaluates the most common linguistic difficulties in children's writing during the first years of education. Participants' perception about their teacher's expectancies towards them was measured through a scale having twenty statements of which eleven were positively and nine negatively stated. Results pointed differences between the students' perception about the expectations towards them, on different groups of difficulty in learning to write. The average perception was lower for the levels of higher difficulty and higher for the groups with less difficulty in learning to write, indicating that the higher the individuals' difficulty learning level was, the less positive was the perception they had about the teachers' expectancies towards them.

Keywords: perception; teachers' expectancies; learning difficulties.

Muitos estudos, sob diferentes enfoques, têm abordado a relação entre variáveis psicológicas e desempenho acadêmico e/ou dificuldades de aprendizagem de alunos. Dentre esses, os que enfatizam a questão da percepção assumem diferentes formas. No que diz respeito ao professor, os estudos têm se centrado na análise de suas percepções, atitudes e expectativas em relação aos alunos, enquanto que as pesquisas envolvendo esses últimos têm se preocupado em investigar a questão de seus sentimentos e crenças, autopercepção, autoconceito, auto-estima e auto-eficácia, por meio da declaração dos mesmos. No que diz respeito à percepção de alunos sobre as expectativas de professores em relação a eles, questão de interesse desta pesquisa, os estudos têm sido bem menos freqüentes. 
A literatura existente sobre as expectativas de professores aponta para a influência que elas podem exercer sobre o desempenho acadêmico de alunos. As pesquisas no campo das expectativas, atitudes e crenças dos professores em sala de aula e seus efeitos têm início na década de 50, mas seu ápice ocorreu na década de 60, mais precisamente em 1968, quando Rosenthal e Jacobson (1968) publicaram a obra "Pigmalião em Sala de Aula", um estudo de grande repercussão. Em seguida, surgiram outros estudos que criticaram essa obra, apontando deficiências que permitiriam dúvidas ao se tirar conclusões sobre os efeitos das expectativas de professores. Durante a década de 70 , continuaram as pesquisas sobre esse tema, das quais Brophy e Good constituem a principal referência teórica. Os trabalhos que investigam essa questão continuaram a ser realizados nas décadas de 80 e 90, apesar de formarem um número mais reduzido.

Em uma revisão de literatura sobre os estudos relativos aos efeitos das expectativas de professores, Brophy e Good (1974) observaram duas grandes categorias de pesquisa. Uma delas envolve trabalhos em que as expectativas foram induzidas experimentalmente, enquanto que a outra contempla estudos em que elas foram naturalmente formadas. No que se refere aos estudos em que as expectativas foram induzidas experimentalmente, nota-se que, quando os sujeitos são professores, o procedimento de indução mais comum é levá-lo a crer em algumas informações falsas que os conduziriam a acreditar que alguns alunos eram mais ou menos capazes do que sugeriam suas capacidades medidas. Em contrapartida, os trabalhos em que as expectativas são naturalmente formadas baseiam-se na situação natural de experiência, sem a influência dos experimentadores, que não fornecem falsas informações ou outros tratamentos artificiais.

O marco do primeiro conjunto de pesquisas sobre os efeitos das expectativas de professores é representado pela obra de Rosenthal e Jacobson (1968). A pesquisa foi feita com o objetivo de verificar o efeito das expectativas dos professores sobre o rendimento dos alunos, especialmente de constatar se os alunos cujos professores tinham maiores expectativas de rendimento eram os que mais realizavam progressos. Para isso, foram apresentadas aos professores as crianças que tinham maior probabilidade de melhorar seu desempenho, tendo sido dito a eles que essas foram as crianças que obtiveram os melhores resultados em um teste de inteligência, quando, na verdade, os alunos foram selecionados aleatoriamente pelos pesquisadores e por seus assistentes. Os resultados revelaram que aquelas crianças que tinham sido indicadas como prováveis a terem melhor desenvolvimento intelectual mostraram um contato mais próximo com os professores, além de serem descritas por esses como as que mais provavelmente teriam sucesso no futuro, como as mais interessadas, como as que apresentavam maior curiosidade intelectual e como as mais felizes.

Em contraste aos estudos cujas expectativas eram induzidas experimentalmente através da apresentação de informações falsas ou através de outros procedimentos experimentais, a década de 70 foi marcada pela superação desse tipo de pesquisas e pela intensificação de estudos naturalísticos sobre efeitos das expectativas de professores. Brophy e Good (1974) constituem a principal referência nessa categoria de estudos, além de serem os responsáveis pela sistematização dos trabalhos nesse campo, fato que os deixa em destaque.

Em uma pesquisa, com o objetivo de utilizar as expectativas geradas naturalmente pelos próprios professores sobre os alunos, solicitou-se aos professores que classificassem seus alunos de acordo com suas próprias expectativas de desempenho. Dessa lista foram selecionados dois grupos de alunos, um com aqueles prováveis a apresentarem alto desempenho e o outro como os que provavelmente apresentariam baixo desempenho. De maneira geral, os resultados mostraram diferenças significativas entre os grupos de alunos divididos com base nas expectativas de seus professores. Aqueles cujos professores manifestaram altas expectativas demonstraram diferentes tipos de contato com seu professor, responderam mais quando perguntas foram feitas, deram mais respostas corretas e poucas incorretas, tiveram poucos problemas nas atividades de leitura e receberam mais elogios e menos críticas do que os alunos julgados como prováveis a apresentarem baixo desempenho (Brophy \& Good, 1974).

Estudos subsequientes investigaram as expectativas de professores sobre os alunos e continuaram apontando para a confirmação de sua influência sobre esses últimos. Babad, Inbar e Rosenthal (1982) realizaram um estudo com o objetivo de verificar os diferentes efeitos das expectativas de professores, que foram influenciados por informações sobre os alunos, e de distinguir os resultados positivos e negativos dessas expectativas. Os professores que foram influenciados por informações negativas sobre alguns alunos os trataram mais negativamente, enquanto que aqueles para os quais as expectativas eram altas foram tratados tão favoravelmente como os alunos que eles mesmos indicaram como tendo alto potencial. Por sua vez, Neves (2002) se preocupou, dentre outras questões, com a análise da existência de relação entre o desempenho de alunos em Matemática e as percepções e expectativas de professores quanto ao seu desempe- 
nho. Os resultados confirmaram essa relação. Aqueles alunos cujos professores apresentaram percepções e expectativas mais favoráveis alcançaram melhor desempenho na disciplina.

Além das expectativas, as percepções e atitudes de professores em relação aos alunos foram investigadas por alguns trabalhos, cujos resultados inferem conclusões semelhantes às indicadas pelos estudos citados, que trataram especificamente da questão das expectativas. Todos os resultados encontrados confirmaram a relação entre tais variáveis e o desempenho de alunos.

No que se refere às percepções de professores sobre os alunos, alguns exemplos são os estudos de Hiebert (1982), Siegel (1992), McLeod (1994), Babad (1995), Meltzer, Katzir-Cohen, Miller e Roditi (2001). De maneira geral, os resultados encontrados revelaram que as percepções de professores sobre os alunos são mais negativas para aqueles indicados por seu baixo desempenho e/ou pelas dificuldades de aprendizagem que apresentam.

A influência das atitudes de professores em relação aos alunos foi investigada por Rolison e Medway (1985), Brigham (1991), Herbert (1992) e Fuchs (1994). Os dados encontrados nesses trabalhos indicaram que as atitudes, que incluem o entusiasmo do professor, suas crenças e as maneiras como planeja e desenvolve suas aulas, são mais negativas para os alunos com menor rendimento acadêmico.

No que se refere ao professor, a literatura existente aponta um número grande de pesquisas que investigaram a influência de suas percepções, atitudes e expectativas em relação ao desempenho e/ou comportamento de seus alunos. Já a questão da percepção de alunos sobre as expectativas, percepções e atitudes de seus professores em relação a eles tem sido bem menos discutida.

Um exemplo de estudo que tratou dessa temática é o trabalho de Davidson e Lang (1960), que teve como objetivo verificar a existência de relação entre a percepção dos alunos sobre o que pensavam ser a opinião de seus professores a respeito deles, a autopercepção, o desempenho acadêmico e o comportamento em sala de aula. As crianças que pontuaram maiores escores de autopercepção foram as que perceberam mais favoravelmente a opinião dos professores em relação a elas. Avaliações positivas dos alunos sobre os sentimentos dos professores em relação a eles se relacionaram tanto com o desempenho acadêmico como com comportamentos desejáveis em sala de aula, isto é, as crianças classificadas como bagunceiras, rebeldes, não-amigáveis mostraram percepções a respeito dos sentimentos dos professores em relação a elas menos favoráveis do que crianças classificadas como zelosas, cooperativas e assertivas.

Nessa mesma direção, o trabalho de Bear, Minke, Griffin e Deemer (1998) investigou os papéis da avaliação positiva percebida sobre o retorno do professor e das comparações sociais do desempenho em leitura nas auto-avaliações de alunos com e sem dificuldades de aprendizagem. Foram avaliados o critério estabelecido pelo aluno para julgamento do sucesso acadêmico, o autoconceito global, a percepção do retorno do professor, a satisfação em leitura e a comparação social do desempenho em leitura. O retorno do professor foi o critério mais comum que os sujeitos apontaram para avaliar seus progressos acadêmicos, tanto em alunos que apresentavam como aqueles que não apresentavam dificuldades de aprendizagem. Além disso, sentimentos de satisfação em leitura, autoconceito global positivo e percepção positiva sobre o retorno do professor estiveram significativamente relacionados em ambos os grupos de alunos. A comparação social também se correlacionou significativamente com essas três variáveis.

Um trabalho nacional realizado por Martinelli e Sisto (2001) também se preocupou em investigar essa avaliação percebida sobre o retorno do professor. Os pesquisadores avaliaram o que alunos com baixo rendimento em escrita acreditavam ser a opinião de seus professores a seu respeito. Os resultados indicaram que quanto mais erros na sua avaliação de escrita as crianças tinham, pior era a sua percepção sobre o julgamento do professor em relação a ela, enquanto que aqueles com melhor desempenho em escrita demonstravam percepções mais positivas.

Ainda que todos esses estudos apontem para a relação entre expectativas de professores e seus efeitos, controvérsias existem sobre essa questão, geradas principalmente pelo estudo de Rosenthal e Jacobson (1968). Vale ressaltar que este gerou muitas discussões no âmbito educacional, levando pessoas a questionarem sobre a real existência desses efeitos. Thorndike (1968) e Snow (1969) criticaram a adequação dos procedimentos de coleta e análise dos dados coletados por Rosenthal e Jacobson (1968) e a apropriação das conclusões feitas com base nessa análise, enquanto que Claiborn (1969) questionou alguns dos seus resultados e sua capacidade de generalização. Apesar disso, a literatura continua apontando para os efeitos das expectativas no comportamento dos indivíduos em geral e, particularmente, sobre a influência que as expectativas exercem sobre a interação professor-aluno e, conseqüentemente, no processo de ensino-aprendizagem. 
Tendo em vista essa polêmica, este trabalho pretende recolocar essa questão como foco de discussão. Nota-se que o estudo das expectativas tem sido freqüentemente analisado partindo-se do professor, de seu comportamento expresso ou verbalizado e de como essas manifestações têm repercutido no comportamento e no desempenho dos alunos. Neste estudo, uma outra análise sobre a influência desse fenômeno será feita, tendo como foco o aluno e não o professor. Além disso, um aspecto do desenvolvimento acadêmico, dificuldades de aprendizagem em escrita, será avaliado para que se possa verificar a relação entre essas duas variáveis.

Para Sisto (2001a), a presença de dificuldade de aprendizagem na escrita geralmente é entendida pela deficiência ao escrever as palavras. O sistema ortográfico é habitualmente tomado como parâmetro de desenvolvimento e pode servir de referência para indicar o processo de apropriação do sistema de escrita.

Nesse sentido, depende em grande parte da concepção de erro, o entendimento sobre as dificuldades que uma criança apresenta para escrever de maneira convencionalmente adequada. Atualmente, compreende-se o erro como inerente ao processo de construção de conhecimentos a respeito da escrita, o que não significa dizer que as questões relativas ao aprendizado da ortografia estejam resolvidas. Se o erro for encarado como etapa do processo de apropriação do sistema ortográfico, a produção escrita da criança pode ser um indício do quanto ela se apropriou do sistema (Zorzi, 1998).

Assim, analisar os erros que um aluno comete para avaliar o seu rendimento pode levar à sugestão de presença ou não de dificuldade de aprendizagem, dependendo de como é feita tal análise. Isso porque existem erros relativamente comuns no início da aprendizagem da escrita, enquanto que a persistência deles após uma longa experiência escolar pode refletir uma dificuldade de aprendizagem.

Segundo Sisto (2001b), há, tradicionalmente, duas maneiras de se interpretar as dificuldades apresentadas por crianças na aprendizagem da escrita, e uma terceira que contempla aspectos de ambas. A primeira delas atribui a dificuldade às deficiências do indivíduo, manifestadas pela predisposição a não aprender determinado conhecimento. A segunda concebe os contextos educativo, familiar e social como responsáveis pela dificuldade, enquanto a terceira acredita na existência de uma interação entre os fatores educativos, familiares, sociais e individuais, sendo que estes últimos apontam para a facilidade ou não dos indivíduos para aprender certos conhecimentos e diferenças na maneira como respondem emocionalmente a situações de tensão e esforço.

Neste estudo será dado enfoque às dificuldades ortográficas, sem a pretensão de avaliar se as dificuldades apresentadas pelas crianças são inerentes ao seu sistema cognitivo, se são produzidas pelo processo de ensino-aprendizagem no qual estão inseridas, ou se são justificadas por ambos os motivos. Dessa forma, o objetivo do trabalho foi verificar se crianças com diferentes níveis de dificuldades em escrita se diferem no que se refere à percepção de expectativas de seus professores a seu respeito.

\section{MÉTODO}

\section{Participantes}

Participaram deste estudo 138 alunos, sendo 72 do sexo masculino e 66 do sexo feminino, entre 9 e 10 anos de idade, freqüentando a terceira série do ensino fundamental, de duas escolas públicas situadas em dois municípios do Estado de São Paulo.

\section{Instrumentos}

1) Escala de Avaliação de Dificuldades de Aprendizagem da Escrita (ADAPE) elaborada por Sisto (2001a), que detecta as dificuldades lingüísticas mais comuns na escrita entre crianças das séries iniciais do ensino fundamental. O texto é constituído por 114 palavras, sendo que 60 delas apresentam algum tipo de dificuldade e 54 não, e que é apresentado à criança em forma de um ditado. As dificuldades estão classificadas como encontro consonantal (tarde, campo, bastante, quando, contaram, festinha, Vanda, aniversário, Amparo, brincadeiras, engraçadas, esparadrapo, jogando, mercúrio, companheiros, Márcio, brincar, crianças, gostam, perto, Jumbo, correndo, gente, Valter, estava, certo, voltar, divertido, pensando, quente, vontade), dígrafos (quando, lhe, festinha, chácara, chegou, machucou, joelho, achou, necessário, passar, burrico, companheiros, Cássio, cachorro, vizinho, chegaram, correndo, quente, velhos, tenho), sílabas compostas (alegre, sobre, brincadeiras, esparadrapo, crianças, brincar, outros, engraçadas, atrás) e sílabas complexas (José, sobre, engraçadas, necessário, seus, Márcio, Adão, crianças, não, sai, gente, difícil, certo, casa, pensando, verão, visitar, aniversário). Além disso, a escala avalia também o uso correto de parágrafos, uso correto da letra maiúscula e acentuação.

Trata-se de um instrumento validado e que se encontra detalhado em Sisto (2001a). Segundo os critérios adotados pelo autor, cada palavra errada é consi- 
derada um item ou unidade de medida. Foi utilizado o critério de classificação de dificuldades de aprendizagem na escrita para a terceira série do ensino fundamental. Segundo esses critérios, até 10 palavras erradas o aluno é considerado como não tendo indícios de dificuldades na escrita, de 11 a 19 palavras erradas considera-se que há uma dificuldade leve, de 20 a 49 erros uma dificuldade média e de 50 ou mais erros uma dificuldade acentuada.

2) Escala de Percepção do Aluno sobre as Expectativas do Professor, elaborada por Martinelli (2005), contendo vinte afirmações, sendo onze positivas e que indicam boa percepção do aluno sobre as expectativas do professor em relação a ele e nove que indicam uma percepção negativa, cujas opções de respostas são sempre, às vezes ou nunca.

Para as questões positivas do instrumento foram atribuídas a pontuação 2 para a resposta sempre, 1 para às vezes e 0 para nunca. Às questões negativas foram atribuídos valores inversos, 0 para a resposta sempre, 1 para às vezes e 2 para nunca, o que revelou uma pontuação bruta para cada sujeito, podendo esta escala variar de 0 a 40 pontos, e indicando que, quanto maior a pontuação obtida, mais positiva é a percepção do sujeito.

\section{Procedimento de Coleta de Dados}

Foi solicitada autorização da direção das escolas depois de apresentar às mesmas os objetivos da pesquisa. Após obter o consentimento dos responsáveis, os instrumentos de avaliação de dificuldades de aprendizagem e de percepção de expectativas foram aplicados coletivamente, pelo professor da classe e pelo próprio experimentador, respectivamente. A aplicação foi feita em oito salas de aula de terceira série do ensino fundamental, em horário regular de aula e teve duração aproximada de 60 minutos em cada turma.

\section{Tratamento estatístico}

A associação entre as duas variáveis foi verificada por meio do índice de correlação de Spearman. O teste de Kruskall-Wallis foi utilizado para verificar a existência de diferenças significativas entre os grupos com dificuldades de aprendizagem em escrita e as percepções de alunos sobre as expectativas do professor a respeito deles. Para identificar a direção ou a localização das diferenças significativas encontradas, aplicouse o teste de Mann-Whitney para amostras independentes. Foi considerado significante todo resultado com p.value (significância) igual ou inferior a 0,05 .

\section{RESULTADOS}

Os resultados estão organizados em dois tópicos. No primeiro serão apresentados os indicadores qualitativos obtidos em relação às duas variáveis investigadas. No segundo, serão apresentadas as associações entre os resultados obtidos nas duas avaliações realizadas.

\section{Dificuldades de aprendizagem em escrita}

Na tabela 1 são apresentados a média e o desviopadrão dos diferentes grupos com dificuldades de aprendizagem em escrita (DA).

Tabela 1. Distribuição dos participantes segundo os grupos de dificuldades em escrita

\begin{tabular}{lccc}
\hline \multicolumn{1}{c}{ Grupos com dificuldades em escrita } & $\mathrm{N}$ & Média & D.P. \\
\hline Grupo 1 - sem indícios de DA & 11 & 7,0 & 2,24 \\
Grupo 2 - DA leve & 27 & 15,19 & 2,59 \\
Grupo 3 - DA média & 79 & 31,42 & 8,67 \\
Grupo 4 - DA acentuada & 21 & 66,05 & 12,65 \\
\hline Total & 138 & 31,57 & 18,74 \\
\hline
\end{tabular}

Observou-se que, dentre os 138 participantes, a maioria deles, quando classificados pelos grupos de dificuldades de aprendizagem em escrita propostos pelo instrumento utilizado (ADAPE), localizaram-se no nível médio de DA (grupo 3), conforme mostra a tabela 1. Isso significa que esse grupo escreveu com erros entre 20 e 49 das palavras que lhes foram ditadas, das 114 do instrumento.

\section{Percepção de alunos sobre as expectativas do professor a seu respeito}

A pontuação obtida com essa escala poderia variar de 0 a 40 pontos, cujo ponto médio é 20 . Os resultados dessa avaliação mostraram que a pontuação obtida pelos participantes variou de 15 a 40 pontos, com média de 29,88 e desvio-padrão de 6,8. Pode-se constatar que a pontuação média encontrada entre os 
participantes se mostrou acima dos 20 pontos, revelando que, de maneira geral, a percepção dos alunos foi mais positiva do que negativa.

\section{Percepção de alunos sobre as expectativas do professor x Dificuldades de aprendizagem}

Quanto à associação entre as variáveis dificuldades de aprendizagem em escrita e percepção de alunos sobre as expectativas do professor, investigada através do coeficiente de correlação não paramétrica de Spearman, encontrou-se uma correlação significativa $(\mathrm{rs}=-0,389, \mathrm{p}<0,0001)$. Esse resultado indica que quanto maior o nível de dificuldade de aprendizagem em escrita dos participantes, menos positiva a percepção que os mesmos tiveram sobre as expectativas de seus professores a seu respeito, ainda que não se possa explicitar uma relação de causalidade entre as variáveis.

A fim de verificar se existiam diferenças entre os grupos com diferentes níveis de dificuldades em escrita e a percepção de expectativas dos mesmos, foi aplicado o teste de Kruskall-Wallis, cujos resultados podem ser observados na tabela 2 .

Tabela 2. Médias de percepção por grupos com dificuldades em escrita

\begin{tabular}{cccc}
\hline $\begin{array}{c}\text { Grupos com dificuldades de } \\
\text { aprendizagem em escrita }\end{array}$ & $\begin{array}{c}\text { Médias em percepção de } \\
\text { expectativas }\end{array}$ & D.P. & $\mathrm{N}$ \\
\hline 1 & 34,36 & 4,25 & 11 \\
2 & 32,93 & 5,36 & 27 \\
3 & 29,25 & 6,06 & 79 \\
4 & 26,00 & 6,27 & 21 \\
\hline Total & 29,88 & 6,28 & 138 \\
\hline
\end{tabular}

Os resultados obtidos através do teste de KruskallWallis mostraram que as diferenças de percepção de alunos sobre as expectativas do professor a seu respeito, verificadas entre os grupos com dificuldades em escrita, são significativas $(\mathrm{p}<0,0001)$.

Nota-se que as médias de percepção de expectativas diminuem conforme aumenta o nível de dificuldade. Para o grupo considerado sem indícios de DA (grupo 1), a média de percepção de expectativas encontrada é a maior entre todos os grupos. A média do grupo de participantes que apresentaram DA leve (grupo 2) é, por sua vez, maior que o grupo de participantes classificados como apresentando DA média (grupo 3), que também é maior que o grupo de participantes com DA acentuada (grupo 4).

Com o objetivo de verificar a direção dessas diferenças, aplicou-se o teste de Mann-Whitney para amostras independentes. Na tabela 3 é apresentada a comparação entre os grupos, discriminando como se revelaram as diferenças estatisticamente significativas.

Tabela 3. Incidência média, Valores de U, Níveis de significância e Comparação entre os grupos com DA em relação à percepção de alunos sobre as expectativas dos professores

\begin{tabular}{ccccc}
\hline $\begin{array}{c}\text { Grupos } \\
\text { com DA }\end{array}$ & $\begin{array}{c}\text { Valores de } \\
\text { U }\end{array}$ & $Z$ & Valores de $p$ & $\begin{array}{c}\text { Comparação } \\
\text { entre os grupos }\end{array}$ \\
\hline G1 e G2 & 126,00 &,- 728 &, 467 & SD \\
G1 e G3 & 218,50 & $-2,665$ &, 008 & G1> G3* \\
G1 e G4 & 31,50 & $-3,340$ &, 001 & G1> G4 \\
G2 e G3 & 684,50 & $-2,774$ &, 006 & G2> G3 \\
G2 e G4 & 110,50 & $-3,602$ &, 000 & G2 $>$ G4 \\
G3 e G4 & 591,00 & $-2,022$ &, 043 & G3 $>$ G4 \\
\hline
\end{tabular}

SD - sem diferenças estatisticamente significantes.

Os resultados obtidos através do teste de MannWhitney indicaram diferenças significativas entre os grupos, com exceção da comparação entre os grupos G1 e G2 de dificuldades de aprendizagem em escrita, que representam respectivamente, alunos sem indícios de DA e DA leve. Nota-se ainda, que o grupo G1 apresenta uma diferença significativa com relação ao grupo G3, representado por dificuldades de aprendizagem médias, e que essa diferença é ainda maior quando comparado ao grupo G4, que inclui as dificuldades de aprendizagem acentuadas. Uma comparação da percepção dos alunos sobre as expectativas do professor permite inferir ainda que a diferença mais 
significante encontra-se entre os grupos G2 e G4, mas que as diferenças também se mostraram significantes entre os grupos G2-G3 e G3-G4.

\section{DISCUSSÃO}

Os estudos abordados neste trabalho referentes às percepções de alunos sobre as expectativas de professores sobre eles retratam a influência que elas podem exercer sobre o comportamento e desempenho acadêmico desses últimos. A obra de Rosenthal e Jacobson (1968) e a contribuição de Brophy e Good (1974) apontaram incisivamente que o comportamento dos professores em sala de aula marca as diferenças na aprendizagem dos alunos. Estudos subseqüentes como os realizados por Babad e cols. (1982) e Neves (2002) continuaram indicando a influência das expectativas dos professores. A influência das atuações do professor, não somente de suas expectativas, mas também de suas percepções e atitudes em relação aos alunos demonstrada nesses estudos, sugere, apesar das controvérsias, uma evidência empírica.

Da mesma maneira, as pesquisas que investigaram a relação entre a percepção de alunos sobre as expectativas de seus professores e o desempenho acadêmico encontraram resultados semelhantes. Os dados da pesquisa realizada por Martinelli e Sisto (2001) sugerem a existência de relação entre percepção do aluno sobre o julgamento do professor a respeito dele e seu desempenho em escrita. Melhor desempenho em escrita demonstrava percepções mais positivas, enquanto que pior desempenho indicava percepções mais negativas. Davidson e Lang (1960) encontraram resultados que mostraram correlações positivas entre percepção de expectativas de professores, autopercepção, desempenho acadêmico e comportamentos desejáveis em sala de aula. Bear e cols. (1998), com a preocupação de investigar a relação entre a avaliação percebida de alunos com e sem dificuldades de aprendizagem sobre o retorno do professor e suas autoavaliações, verificaram que o retorno do professor foi apontado pelos sujeitos como o critério mais comum para avaliarem seus progressos acadêmicos para ambos os grupos.

Ainda que nenhum desses trabalhos tenha identificado a relação de causalidade entre as percepções e o desempenho acadêmico e/ou as dificuldades de aprendizagem, relações entre essas variáveis foram verificadas. Isso pode sugerir a influência que exercem as interpretações pessoais dos indivíduos acerca de diversos assuntos. Baseada nessa evidência, esta pesquisa partiu desse referencial teórico, que indica a existência desse fenômeno, preocupando-se em verificar como o aluno percebe tais expectativas e como esta percepção se relaciona com a dificuldade de aprendizagem em escrita.

Os dados encontrados nessa pesquisa são similares aos resultados desses três trabalhos. Os resultados revelaram que a percepção de alunos sobre as expectativas de seus professores a respeito deles mostrou-se relacionada às dificuldades de aprendizagem em escrita. Quanto maior o nível de dificuldade, menos positiva se mostrou a percepção que os alunos têm sobre as expectativas dos professores. Ainda que não se possa afirmar que tal percepção é a responsável pelas dificuldades que os sujeitos apresentaram, e nem o contrário, é possível dizer da relação entre as duas variáveis.

Nota-se, ao analisar esses dados, um paradoxo interessante. Maiores dificuldades na escrita indicaram percepções de expectativas menos positivas. A comparação entre os grupos mostrou que as diferenças foram mais significativas entre os grupos $\mathrm{G} 2$ e G4, o que nos permite inferir que, à medida que as dificuldades começam a se acentuar, as percepções negativas dos alunos sobre as expectativas de seus professores começam a se intensificar. No entanto, é interessante observar que não foram estatisticamente significativas as diferenças entre os grupos G1 e G2, que representam respectivamente ausência de dificuldades e indício de dificuldade leve, embora tenha se notado que o G1 tenha uma percepção mais positiva que o G2. Acredita-se que como as dificuldades ainda não estão muito demarcadas entre esses dois grupos, tanto os professores quanto os alunos podem não ter tomado consciência delas, não interferindo na percepção de ambos.

Ainda que os resultados desses estudos sejam muito interessantes, há que se considerar que existem alguns questionamentos a serem feitos ou dificuldades que merecem serem discutidas em estudos dessa natureza. Uma questão a ser analisada é a congruência entre as percepções de alunos e as expectativas de professores. Não se pode afirmar que a percepção do aluno sobre as expectativas de seus professores indique as reais expectativas desses professores. Se por um lado não é possível afirmar que a percepção dos alunos quanto às expectativas dos professores a seu respeito indique as reais expectativas destes, por outro, é pertinente considerarmos, como afirmam Coll e Miras (1995), que nossas atitudes em relação aos fenômenos sociais e às outras pessoas estão em grande parte mediadas pela percepção e pela representação que temos delas. Assim, a percepção de alunos sobre os professores e destes sobre os alunos tem seu papel em suas vidas escolares, mesmo porque, para o aluno, 
a expectativa que o professor tem a seu respeito é a que ele percebe, ou seja, o que o influencia é a sua interpretação sobre as expectativas dos professores.

Segundo Leite (1981), na situação de sala de aula, assim como em todas as outras situações da vida humana, é quase impossível o professor manter uma atitude de neutralidade para com seus alunos. Quase sempre deixam passar, geralmente de modo inconsciente, suas preferências ou antipatias, que são percebidas pelos alunos. A qualidade percebida, pelo fato de ter sido percebida, tende a ser acentuada. Cooper (1979) diz que, embora seja difícil estabelecer relações causais entre as expectativas do professor e o bom ou mau desempenho dos alunos, é possível afirmar que elas têm a força de manter o desempenho do aluno em um nível desejável ou não. Esse fato coloca em destaque a importância desse fenômeno que, segundo o autor, deveria se constituir em objeto de preocupação não somente das pessoas ligadas à educação, mas de toda a sociedade.

De acordo com Davidson e Lang (1960), os incentivos básicos que um professor pode oferecer aos seus alunos, para que aprendam com sucesso, são a sua aceitação, de um lado, e sua aprovação, de outro. Os sentimentos do professor de aceitação e de aprovação são comunicados ao indivíduo e percebidos por ele como positivos. É provável que avaliações como essa encorajem o aluno a procurar cada vez mais a aprovação de seu professor, através de seu bom desempenho e bom comportamento. Da mesma maneira, esse ciclo pode ser iniciado pelo aluno, que, comportando-se bem, agradará o seu professor que, por sua vez, comunicará sentimentos positivos, reforçando seu desejo de continuar sendo um bom aluno. Contudo, como enfatizam esses pesquisadores, é difícil dizer qual dessas variáveis determina a outra, mas uma implicação é clara: a importância de um professor comunicar sentimentos positivos em relação aos seus alunos, que fortaleçam a percepção de si mesmos e estimulem seu crescimento acadêmico assim como interpessoal.

Uma outra questão a ser discutida é a dificuldade encontrada nas ciências humanas em geral e também nesta área de pesquisa referente à mensuração de variáveis que são psicológicas e, por isso, subjetivas. Thorndike (1968), Snow (1969) e Claiborn (1969) criticaram o instrumento utilizado por Rosenthal e Jacobson (1968), assim como os procedimentos de coleta, de análise dos dados, de alguns resultados e generalizações feitas pelos autores. Da mesma maneira, esses mesmos critérios são muitas vezes criticados na área de psicologia, dada a dificuldade mencionada. Por se tratar de aspectos subjetivos, há que se considerar que algumas variáveis possam interferir nas res- postas dos indivíduos, que talvez respondam tendo em vista o que imaginam ser mais aceito por outros. Há também que se considerar que, neste tipo de estudo, não se pode inferir relações de causa e efeito entre as variáveis avaliadas, o que não permite que a compreensão do fenômeno ocorra de maneira mais abrangente.

Para finalizar cumpre destacar que os achados deste estudo são mais uma sugestão do que uma conclusão a ser aplicada a qualquer contexto e de maneira geral, já que cada interação entre professor e alunos implica peculiaridades específicas. Atua, entretanto, como contribuição no sentido de ser uma informação, um dado a mais disponível sobre esse tema. Dados que poderão, talvez, ser reconhecidos e reinterpretados por outros professores em seus contextos de sala de aula. A investigação desse aspecto da interação professor-aluno, a percepção de alunos sobre as expectativas de seus professores, pode contribuir para a construção de conhecimentos pertinentes ao processo ensino-aprendizagem e propiciar discussões que venham a trazer melhorias em salas de aula, ao mesmo tempo que constitui um dado importante para a análise desse processo.

\section{REFERÊNCIAS}

Babad, E. Y. (1995). The teacher's pet phenomenon, student's perceptions of teacher's differential behavior, and students' morale. Journal of Educational Psychology, 87, 361-374.

Babad, E. Y., Inbar, J. \& Rosenthal, R. (1982). Pygmalion, galatea, and the golem: Investigations of biased and unbiased teachers. Journal of Educational Psychology, 74, 459-474.

Bear, G. C., Minke, K. M., Griffin, S. M. \& Deemer, S. A. (1998). Achievement-related perceptions of children with learning disabilities and normal achievement: group and developmental differences. Journal of Learning Disabilities, 31(1), 91-104.

Brigham, F. J. (1991). Generating excitement: teacher enthusiasm and students with learning disabilities. Paper presented at the Annual Meeting of the Council for Learning Disabilities (Minneapolis, MN, October 10-12). Obtido em 17 de outubro de 2002, do ERIC database no World Wide Web: http:// www.eric.ed.gov.

Brophy, J. E. \& Good, T. L. (1974). Teacher-student relationships: cause and consequences. New York: Holt, Rinehart and Winston.

Claiborn, W. (1969). Expectancy effects in the classroom: a failure to replicate. Journal of Educational Psychology, 60, 377383.

Coll, C. \& Miras, M. (1995). A representação mútua professor/aluno e suas repercussões sobre o ensino e a aprendizagem. Em C. C. Coll, J.Palácios \& A. Marchesi (Orgs.), Desenvolvimento psicológico e educação, v. 2 (pp. 265-280). Porto Alegre: Artes Médicas.

Cooper, H. M. (1979). Pygmalion growsup: a model for teacher expectation communication and performance influence. Review of Educational Research. Washington, 49(3), 389-410. 
Davidson, H. \& Lang, G. (1960). Children's perceptions of their teachers' feelings toward them related to self-perception, school achievement and behavior. Journal of Experimental Education, 29, 107-118.

Fuchs, L. S. (1994). The relation between teachers' beliefs about the importance of good student work habits, teacher planning, and student achievement. Elementary School Journal, 94(3), 331-345.

Herbert, J. M. (1992). Intervening to influence teachers' actions and students' involvement. Journal of Personnel Evaluation in Education, 6(3), 249-261.

Hiebert, B. (1982). Affective influences on learning disabled adolescents. Learning Disability Quarterly, 5(4), 334-343.

Leite, D. (1981). Educação e relações interpessoais. Em M. H. S. Patto (Org.), Introdução à psicologia escolar ( $2^{\mathrm{a}} \mathrm{ed}$.) (pp. 234257). São Paulo: T. A Queiroz.

Martinelli, S. C. (2005). Construção de uma medida de avaliação da relação professor-aluno. Em Instituto Brasileiro de Avaliação Psicológica (Org.), Anais do II Congresso Brasileiro de Avaliação Psicológica (CD Room). Gramado: RS.

Martinelli, S. C. \& Sisto. F.F. (2001). Avaliação da relação professor-aluno: O olhar do aluno em fase da aquisição da escrita. Em Sociedade Brasileira de Psicologia (Org.), Anais da XXXI Reunião Anual de Psicologia (pp. 212-213). Ribeirão Preto: SBP.

McLeod, T. M. (1994). Social skills, school skills, and success in the high school: a comparison of teachers' and students' perceptions. Learning Disabilities Research and Practice, 9(3), 142-147.

Meltzer, L., Katzir- Cohen, T., Miller, L.\& Roditi, B. (2001). The impact of effort and strategy use on academic performance: student and teacher perceptions. Learning Disability Quarterly, 24(2), 85-98.

Neves, L. F. (2002). Um estudo sobre as relações entre a percepção e as expectativas dos professores e dos alunos e o desem- penho em matemática. Dissertação de Mestrado, Faculdade de Educação, Universidade Estadual de Campinas. Campinas, SP.

Rolison, M. A. \& Medway, F. J. (1985). Teachers' expectations and attributions for student achievement: effects of label performance pattern, and special education intervention. American Educational Research Journal, 22(4), 561-573.

Rosenthal, R. \& Jacobson, L. (1968). Pygmalion in the classroom: teacher expectation and pupil's intellectual development. New York: Holt, Rhinehat \& Winston.

Siegel, J. (1992). Teachers' attitudes toward their integrated learning handicapped students: Relation to teacher perceptions of students' behavior. Obtido em 30 de outubro de 2002, do ERIC database no World Wide Web: http:// www.eric.ed.g.

Sisto, F. F. (2001a). Dificuldades de aprendizagem em escrita: um instrumento de avaliação (Adape). Em F. F. SISTO, E. Boruchovitch, L. D. T. Fini, R. P. Brenelli, S. C.Martinelli (Orgs.), Dificuldades de aprendizagem no contexto psicopedagógico (pp. 190-213). Petrópolis: Vozes.

Sisto, F. F. (2001b). Dificuldades de Aprendizagem. Em F. F. Sisto, E. Boruchovitch, L. D. T. Fini, R. P. Brenelli, S. C. Martinelli (Orgs.), Dificuldades de aprendizagem no contexto psicopedagógico (pp. 19-39). Petrópolis: Vozes.

Snow, R. E. (1969). Unfinished pygmalion. Contemporary Psychology, XIV(4), 197-199.

Thorndike, R. (1968). Review of pygmalion in the classroom. American Educational Research Journal, 5, 708-711.

Zorzi, J. L. (1998). Aprender a escrever: a apropriação do sistema ortográfico. Porto Alegre: Artes Médicas.

Recebido: $22 / 08 / 2005$

Revisado: 14/10/2005

Aceito: 05/12/2005

\section{Sobre as autoras:}

Andreza Schiavoni: Doutoranda na área de Psicologia Educacional da Universidade Estadual de Campinas, Professora da Universidade Federal de Uberlândia (andrezaschiavoni@ hotmail.com)

Selma de Cássia Martinelli: Professora Doutora do Departamento de Psicologia Educacional da Universidade Estadual de Campinas (selmacm@unicamp.br)

Endereço para correspondência: Faculdade de Educação - Departamento de Psicologia Educacional - UNICAMP, Rua Bertrand Russell, 801, Barão Geraldo - Campinas/SP, CEP 13083-970. 